\title{
EL COMERCIO VENECIANO DE MATERIAL IMPRESO Y PAPELERÍA CON ESPAÑA: LOS PEDIDOS DE JOSEP BRO, LIBRERO DE GIRONA, A LA CASA REMONDINI (1764-1775)
}

\author{
JAVIER ANTÓN PeLAyo \\ Universitat Autònoma de Barcelona
}

\begin{abstract}
RESUMEN. En la primera mitad del siglo XVIII los libreros españoles abastecieron sus tiendas con obras provistas por libreros flamencos, franceses y suizos. Después de mediados de siglo, y sobre todo durante los años sesenta y setenta del siglo XVIII, un número notable de libreros y comerciantes, asentados mayormente en ciudades mediterráneas, establecieron relaciones con impresores de la república de Venecia. Entre los impresores venecianos destacó la casa Remondini, la cual consiguió introducir en la Península Ibérica libros, estampas y papeles pintados con notable éxito. En este artículo se analiza la relación comercial entre Josep Bro, librero de Girona, y la casa Remondini, un ejemplo paradigmático de la naturaleza de estos vínculos.
\end{abstract}

Palabras clave: Josep Bro, Remondini, Venecia, Girona, imprenta, librería, siglo XVIII, comercio.

AbstraCt. In the first half of the XVIIIth century, the Spanish booksellers supplied their shops with works bought to Flemish, French and Swiss booksellers. After the middle of that century, and especially during the sixties and seventies of the XVIIIth century, a notable number of booksellers and merchants, seated mainly in Mediterranean cities, established relations with printers of the republic of Venice. Between the Venetian printers, stood out the house Remondini, which managed to introduce in the Iberian Peninsula books, prints and papers painted with notable success. In this article, the author analyzes the commercial relation

Recibido: 30-3-2017 • Aceptado: 31-5-2017 • Javier.Anton@uab.cat

Mi más sincero agradecimiento al profesor Mario Infelise (Università Ca'Foscari. Venecia), que tuvo la amabilidad de revisar este texto y sugerirme sabios consejos. 
between Josep Bro, bookseller of Girona, and the house Remondini. This is a paradigmatic example of the nature of these links.

Key words: Josep Bro, Remondini, Venice, Gerone, print, library, Eighteenth Century, trade.

\section{Introducción}

Entre los diferentes aspectos de análisis de la historia del libro en España, el comercio ha resultado ser un ámbito difícil de abordar y, particularmente, la importación de libros impresos fuera del Reino. La carencia de archivos privados de libreros y mercaderes de libros y, por consiguiente, la casi ausencia de registros contables y correspondencia, ha supuesto un inconveniente a la hora de conocer en profundidad su relación con los grandes centros editoriales europeos: la naturaleza de las mercancías compradas, el coste de los productos, las estrategias comerciales, las rutas empleadas y el beneficio obtenido (Rey, 2008, pp. 277-280; Bertrand, 2016).

La bibliografía que aborda este tema ha buscado alternativas en fuentes indirectas, como los protocolos notariales, los catálogos de los libreros y la procedencia de los volúmenes alojados en las bibliotecas privadas e institucionales. Las noticias que arrojan los fondos notariales, aunque dispersas y difíciles de localizar, acostumbran a aportar informaciones originales e inéditas (García Cuadrado, 2015); los catálogos de los libreros, a pesar de su condición efímera y su escasez, «constituyen una fuente de primer orden para el estudio de la circulación del libro y las redes de distribución» (Rueda, 2012, p. 196; Bas, 2013; Rueda y Agustí, 2016); y el análisis de las bibliotecas privadas o públicas, en donde a menudo se detecta un claro dominio de ediciones forasteras, pone en evidencia la existencia de un activo comercio de libros todavía no evaluado en toda su dimensión (Arias de Saavedra, 2003 y 2017; Rey, 2008; Antón, 2011).

Además de estas fuentes, abundantes referencias a estas relaciones comerciales se hallan asentadas en los fondos documentales que de estos importantes impresores y libreros conservan los archivos suizos, franceses o italianos ${ }^{1}$. En el caso veneciano, destaca el fondo documental de la familia Remondini, depositado en el Museo Civico de Bassano del Grappa que, entre otros materiales, reúne cerca de 7.000 cartas dirigi-

En Suiza, los archivos de la Société Typographyque de Neuchâtel (1769-1798) (STN Online Database Archive); los Archives d'Etat (AEG), Ginebra, que custodian el «Grand livre» de los hermanos Cramer (1755-1767), Com. F 57 y las «Copies de lettres» de los hermanos Gosse (1759-1791), Com. F 61-63. En Francia, la Collection Anisson-Duperron sur la librairie et l'imprimerie, en la Bibliothèque Nationale de France (BNF), recoge correspondencia de los hermanos de Tournes con la Dirección de la Librería de París y de los libreros François Grasset y Antoine Boudet. 
das a los impresores y libreros de Europa entre finales del siglo XVII y principios del siglo XIX. De entre la nómina de corresponsales españoles, para este artículo se han tenido en cuenta las misivas remitidas por Oleguer Verdaguer y Ros, el agente que veló por los intereses de la casa Remondini en Cataluña entre 1768 y $1780^{2}$.

Por otro lado, la documentación judicial relativa al intercambio de material impreso ofrece la extraordinaria posibilidad de poner al descubierto los procedimientos comerciales y los mecanismos que se aplicaban cuando se había perdido la seguridad en los tratos. A través de la causa judicial que la casa Remondini emprendió en 1782 ante el Tribunal del Consulado de Comercio de Barcelona contra su deudor, el librero de Girona Josep Bro, ha sido posible analizar los pedidos realizados, el coste de las mercancías suministradas, las rutas empleadas, los tiempos de demora, los sistemas de pago y la gestión de los impagos ${ }^{3}$.

Esta documentación, sin embargo, muestra toda su excelencia cuando se ensambla con la copiosa información disponible sobre los actores implicados y sus negocios editoriales y librarios. La vida profesional y personal del impresor y librero gerundense Josep Bro ha sido trazada por Enric Mirambell (1988) y Javier Antón (1998), aunque este estudio, además, se ha enriquecido con la búsqueda sistemática en los fondos notariales de la ciudad de Girona. Por otro lado, la historia de la casa Remondini de Bassano, seguramente el negocio editorial más grande de la Europa de su tiempo, ha sido objeto de numerosos estudios, entre los cuales hay que destacar los trabajos e iniciativas impulsadas de Mario Infelise y Paola Marini (Infelise, 1990 y 2016; Infelise y Marini, 1990 y 1992).

\section{La importación de libros en España}

A tenor de las investigaciones realizadas sobre la importación de libros en España durante el siglo XVIII (Bonnant, 1999, pp. 31-56; Péligry, 1984; López, 2003), se puede deducir que, durante la primera parte de la centuria, como consecuencia del estado decadente de la imprenta española y la inobservancia de la antigua legislación relativa a este ramo, la entrada de libros y material impreso apenas encontró obstáculos, más allá de la vigilancia — bastante disminuida — de la Inquisición (Caro, 2004). Los impresores-libreros flamencos, franceses, suizos e italianos inundaron el mercado español de un variado surtido de mercancías editoriales: obras en romance de autores españoles; libros litúrgicos, legales y científicos, normalmente latinos; y estampas

Archivio Remondini depositado en el Museo Civico di Bassano del Grappa (MCBG-AR), 9.

Archivo de la Corona de Aragón (ACA), Real Audiencia, Consulado y Tribunal de Comercio, C 6806, «Causa de José Remondiny e hijos (sociedad) (Venecia, Italia) contra Bro, José, impresor de Girona» (1782) 
finas y populares. Todos estos materiales se fabricaban en el extranjero con diligencia, excelente calidad y eran vendidos a precios muy ventajosos en España. De la distribución se hicieron cargo, por un lado, los propios editores (Deville, Tournes, Cramer, Grasset o Boudet), los cuales se esforzaron por mantener correspondencia con los libreros, mercaderes de libros y eruditos españoles (Mestre, 1984); y, por otro, un nutrido grupo de libreros/quincalleros originarios mayoritariamente del Delfinado, los cuales, instalados en la Península Ibérica, sobre todo en las ciudades del Mediterráneo y Portugal, mantuvieron estrechas relaciones comerciales con los grandes centros editoriales franceses y suizos (Piwnik, 1989; Fontaine, 1993).

Esta libertad en la importación de libros del extranjero quedó aminorada durante la década de los cincuenta del siglo XVIII. En noviembre de 1752 un auto del juez de imprentas Juan Curiel estableció con inusitado rigor la prohibición de que «ningún librero o tratante en libros ni otra alguna persona pueda vender o meter en estos reinos libros ni obras de romance compuestas por los naturales de estos reinos e impresos fuera de ellos sin expresa real licencia de S.M., so pena de muerte y de perdimiento de bienes». Además de este célebre punto trece del auto, también otras cláusulas ponían trabas a la importación de libros: el punto catorce ordenaba que los libros importados del extranjero tenían que ser tasados por el Consejo de Castilla ${ }^{4}$; el punto quince establecía que, sin licencia especial, no se podían vender los libros escritos por extranjeros de primera impresión y por naturales de segunda impresión fuera del reino; el punto dieciséis pedía la colaboración de los libreros en las visitas que podían realizar a sus tiendas los subdelegados del superintendente de imprentas; y el punto dieciocho exigía el examen de los libros de coro (misales, breviarios, diurnales, pontificales), escritos en latín o en romance, impresos fuera del reino (Escolano, 1796, pp. 416-421; Bragado y Caro, 2004, pp. 577-579; De los Reyes, 2000, I, pp. 477-515).

Aunque los libreros de Madrid recurrieron el auto de Curiel la norma fue ratificada casi en su totalidad en junio de 1754. Dos años después, el librero Francisco de Mena, uno de los cabecillas de la protesta, escribía al librero ginebrino François Grasset dando cuenta de la difícil situación que se avecinaba para los libreros españoles, «qui ruine et détruit totalement notre commerce et, par consequent, le vôtre» (López, 1987, pp. 398-399). Efectivamente, los grandes impresores y libreros de Lyon, Ginebra, Lausana y Venecia también se alarmaron por el decreto de Curiel.

La tasación de los libros para su venta fue abolida por la Real Orden de 14 de noviembre de 1762. A partir de entonces, salvo unos cuantos libros destinados a la instrucción y la devoción popular, se estableció la libertad para fijar el precio de venta de los libros. 
Las disposiciones legislativas tomadas en España contribuyeron a alterar los flujos comerciales de material impreso, aunque también las propias vicisitudes de algunos centros editoriales europeos facilitaron los cambios que experimentó el mercado internacional de libros y estampas. A pesar que todavía los datos disponibles son insuficientes, parece que durante los primeros cincuenta años del siglo XVIII los libreros de los Países Bajos, Francia y Suiza mantuvieron una dura competencia por el dominio del mercado ibérico. A finales de la década de los cincuenta, sin embargo, el tráfico librario de estos países con España se estancó o disminuyó. Georges Bonnant, por ejemplo, sugiere que la época de comercio más intenso de Ginebra con la Península Ibérica fue el período 1730-1760 (Bonnant, 1999, p. 52). Por contra, los editores italianos, sobre todo venecianos, llevaron a cabo desde la cuarta década del Setecientos una intensa estrategia comercial que les permitió dominar el mercado hasta finales de la década de los setenta de la centuria. Mario Infelise, empleando los registros de los «dazi» (derechos aduaneros) de los V Savi alla Mercanzia, la institución que se encargaba de controlar el comercio marítimo de Venecia, concluye que el quinquenio 1750-1755 fue el período algido de las exportaciones de libros destinados a la Península Ibérica; el período 1755-1770 permanece ausente de datos; y durante las dos décadas siguientes, hasta 1790, se mantuvo una notable actividad exportadora (Infelise, 1992, pp. 217-261).

El retrato más preciso que tenemos sobre la participación de los editores europeos en el mercado del impreso en España es la estimación que el librero parisino Antoine Boudet realizó en 1763 cuando viajó por ella. Según sus cálculos, el volumen de este negocio para España alcanzaba las 750.000 libras tornesas, las cuales se repartían de la siguiente manera: Avignon, 50.000 libras; Roma, Milán y Luca, 50.000 libras; Lausana, Ginebra, Lyon, París, 100.000 libras; Amberes, 200.000 libras; y Venecia 350.000 libras (Guinard, 1957). La aportación veneciana suponía casi el $47 \%$ del total, lo cual manifiesta un claro dominio de Su Serenísima en el comercio del libro con España. La casa Remondini fue la firma editorial más preeminente de la república de Venecia que envió material impreso a la península y Josep Bro, librero de Girona, uno de sus clientes más humildes e informales.

\section{Josep Bro, impresor y librero de Girona, y la casa Remondini de Venecia}

Josep Bro fue el representante más destacado de una estirpe de impresores que trabajó en la ciudad de Girona durante el siglo XVIII. Originarios del sur de Francia, los hermanos Gabriel y Jaume Bro se establecieron en Girona en los primeros años del Setecientos. Josep Bro se formó en el taller de su padre Jaume y, en 
1749, ingresó en el gremio de San Jerónimo que reunía a los impresores de la ciudad. Al año siguiente se separó de su padre y estableció un taller propio, iniciando una intensa actividad tipográfica. Como era habitual entre los impresores gerundenses del siglo XVIII, también poseía un establecimiento de librería aunque, paralelamente, se dedicaba a otras actividades ajenas a su profesión, como las de empleado municipal, empresario de la construcción o gestor de sus propiedades.

Desde 1750 hasta 1794 — fecha de su muerte — publicó una treintena de libros, pero imprimió muchísimos bandos, órdenes reales, edictos, memoriales, listas de precios, plantillas administrativas, programas de fiestas, currículums, sermones, comedias, impresos forenses o anuncios públicos. El hecho de ser impresor real desde 1752 le reportó contínuos encargos por parte de las administraciones.

Como librero, entre 1751 y 1755, tuvo la exclusiva en la ciudad de Girona para vender los libros que, destinados a la enseñanza, tiraba la imprenta de la Univesidad de Cervera; tenía tratos con diversos impresores y libreros de Barcelona; y se abasteció de libros, estampas y material de papelería procedentes de Venecia entre 1764 y 1775. Disponía de una tienda en la calle Ballesterias que, a su muerte, reunía unos 12.000 volúmenes y 107 resmas de papel estampado.

Por su parte, la casa Remondini se estableció en Bassano (Veneto, Italia) a mediados del siglo XVII. En un principio, más que a la producción de libros, se dedicó a la impresión de estampas religiosas y pequeños libros de devoción, una mercancía de mediana calidad pero servida a precios muy asequibles.

En 1725, Giuseppe Remondini amplió y racionalizó el proceso productivo, alquilando tres molinos de papel y fundando una escuela de grabadores. Tras la muerte de Giuseppe en 1742 estuvieron al frente del negocio tipográfico su hijo Giambattista y, luego, su nieto Giuseppe, los cuales llevaron la empresa hasta su máximo esplendor. Se impulsó la producción de libros — más de 30 títulos al añoy se mejoró la impresión de estampas. La empresa editorial se organizó de forma vertical, orquestando una estructura productiva completa que permitió una notable reducción en los costos. Por todo ello, cuando Giambattista murió en 1773, dejó la imprenta más grande de la república véneta y, posiblemente, la más grande de Europa. Las 54 prensas que tenía a su disposición (32 para estampas «in rama», 18 para libros y 4 para papel dorado), los molinos de papel, la fundición de tipos y los talleres para la elaboración de grabados empleaban a un millar de personas.

Para la difusión de la copiosa mercancía que producía la imprenta se utilizó a centenares de vendedores ambulantes del valle del Tesino y se organizó un sistema de distribución capilar que implicaba la participación de decenas de corresponsales establecidos por toda Europa, desde Polonia hasta Portugal. Además, a partir de 1729 
los Remondini editaron catálogos que describían los productos, los precios y las condiciones de venta (Carraro, 2013).

\section{Los pedidos de Josep Bro a la imprenta Remondini de Venecia}

Josep Bro realizó cuatro pedidos de libros, encuadernaciones, estampas y material de papelería a la casa Remondini: en 1763, 1768, 1773 y $1775^{5}$. El impresor y librero gerundense compraba a partir de los catálogos de venta que la empresa de Bassano ponía a disposición de sus clientes. En estos catálogos se describía el género y se anotaban los precios. En las facturas, sin embargo, se aplicaban una serie de descuentos que, por norma, eran del $30 \%$ para los libros impresos por la propia casa; del $20 \%$ para los libros impresos por otros impresores venecianos; del $10 \%$ para los libros de uso eclesiástico - como biblias, breviarios, martiriologios, oficios, rituales-y para las estampas y el papel decorado. A las encuadernaciones, por contra, no se aplicaba ningún tipo de rebaja.

Las facturas también sumaban los gastos de embalaje y el seguro del transporte por mar cuando se empleaba este medio para llevar la mercancía hasta Génova, la plaza comercial que actuaba de intermediaria del tráfico entre Venecia y España. Los fardos o cajas que custodiaban el género - marcadas siempre con las iniciales G. B., Giuseppe Bro - tuvieron un precio de entre 16 y 25 libras venecianas, cantidades que apenas representaban el $1 \%$ del coste de cada envío. El seguro que se suscribía significaba el $2 \%$ del valor del material transportado y corría a cargo del cliente, pero del coste de la conducción del material hasta Génova se hacía cargo la casa Remondini. Luego, desde Génova hasta la costa catalana, el comprador asumía los gastos del transporte.

Sumando los precios de todos los géneros solicitados y el valor de los portes y seguros de mar $-\mathrm{y}$ restando los descuentos aplicados - el librero gerundense adquirió mercancías por valor de 7.913 libras venecianas (1.483 libras, 13 sueldos y 9 dineros, en moneda barcelonesa o catalana ${ }^{6}$. El resumen contable de la facturación es el siguiente:

ACA, Real Audiencia, Consulado y Tribunal de Comercio, C 6806, «Causa de José Remondiny... contra Bro» (1782).

6 Cada libra veneciana, según el cambio corriente en aquellos años, correspondía a 3 sueldos y 9 dineros en moneda catalana. 
Tabla 1. Facturación de la imprenta Remondini de Venecia a Josep Bro, librero de Girona (1764-1775)

\begin{tabular}{|c|c|c|c|c|c|c|}
\hline FACTURAS & Tomos & $\begin{array}{r}\text { Encuadernaciones } \\
\text { coste }\end{array}$ & $\begin{array}{l}\text { Estampas/papeles } \\
\text { coste }\end{array}$ & Gastos envío & Gastos seguro & $\begin{array}{l}\text { COSTES } \\
\text { TOTALES }\end{array}$ \\
\hline Fac. 1 & 118 & $141^{[1]}$ & $\begin{array}{l}1.425 \text { estampas } \\
4,75 \text { resmas papel }\end{array}$ & & & \\
\hline 1764,28 julio & 1.120.11 & 327.10 & 381.06 & 17.03 & 35.10 & 1.882 .00 \\
\hline $\begin{array}{l}\text { Fac. } 2 \\
1768,19 \text { noviembre }\end{array}$ & 1.658 .08 & - & $\begin{array}{l}- \\
-\end{array}$ & 16.12 & - & 1.675 .00 \\
\hline $\begin{array}{l}\text { Fac. } 3 \\
1773,4 \text { septiembre }\end{array}$ & 1.799 .04 & - & $\begin{array}{l}4,5 \text { resmas papel } \\
252.00\end{array}$ & 20.16 & 41.00 & 2.113 .00 \\
\hline $\begin{array}{l}\text { Fac. } 4 \\
1775,14 \text { octubre }\end{array}$ & 2.147 .18 & 70.10 & - & 24.12 & - & 2.243 .00 \\
\hline $\begin{array}{l}\text { Total mercancías } \\
\text { Total costes }\end{array}$ & 6.726 .01 & 398.00 & $\begin{array}{l}1.425 \text { estampas } \\
9,25 \text { resmas papel } \\
\end{array}$ & 79.03 & 76.10 & 7.913 .00 \\
\hline
\end{tabular}

[1] Nótese que el número de encuadernaciones supera el número de tomos. Es probable que algunos tomos se encuadernasen en más de un volumen.

* Elaboración propia a partir de ACA, Real Audiencia, Consulado y Tribunal de Comercio, C 6806

* Las cantidades están dadas en libras y sueldos, moneda veneciana.

El $85 \%$ de la la facturación hacía referencia a la compra de libros, el $8 \%$ a la adquisición de estampas y papeles decorados, el $5 \%$ a encuadernaciones y el $2 \%$ a los gastos de envío y seguro de mar.

Todas las obras encargadas eran latinas y trataban aspectos religiosos en sentido amplio y, en mucha menor medida, médicos. Uno de cada tres ejemplares solicitados iban destinados para uso de eclesiásticos — sobre todo misales_- y también se incluían decenas de volúmenes teológicos, como la Summa de Noël Alexandre, la Theología de François Genet, el Tribunal confessariorum de Martín Wigandt, las obras del Papa Benedicto XIV, la Theología de Fulgencio Cuniliati y el Annus apostolicus de Zacarías Laselve. Los libros de medicina estaban representados en dos docenas de tomos, siendo el autor más demandado el cientifico italiano Giovanni Battista Morgagni.

Los libros del primer pedido fueron encuadernados en su mayor parte a la holandesa y una pequeña parte en cartón, mientras que los libros de los pedidos posteriores fueron remitidos sin encuadernar, a excepción de 24 misales y diurnos que fueron forrados con una cierta suntuosidad. De hecho, en la encuadernación es donde los libreros podían conseguir un amplio margen de ganancia.

Las estampas sólo se solicitaron al principio pero la remesa incluía una variada gama del surtido que ofrecía la casa de Bassano: imágenes de crucifijos, vírgenes, santos y santas, escenas domésticas, personajes típicos, vedute urbanas, paisajes, 
animales y plantas; podían ser en negro o miniadas con diversos colores; y de diferentes tamaños, según fueran imperiali, reali, realetti, francesine, chinesi o santi da breviario. También los papeles encargados recogían una amplia gama de la oferta de los Remondini: dorado, colorado o de indiana — decorados con flores, animalillos o figurillas - y marmolado, conchado o salpicado.

\section{Las normas comerciales de la casa Remondini. Los pagos e impagos de Josep Bro}

El surtido de material impreso y papelería que ofrecía la casa Remondini era muy amplio y variado. El principal mecanismo para dar a conocer su mercancía entre los posibles clientes era a través de unos detallados catálogos que empezaron a publicar a partir de 1729 (Carnelos, 2008). Durante la segunda mitad del siglo XVIII pusieron a disposición de la clientela 27 catálogos impresos, incluyendo apéndices, suplementos y repertorios especializados (Infelise y Marini, 1990, pp. 77-81). En el período durante el cual Josep Bro realizó sus pedidos (1763-1775), pudo tener acceso a los catálogos de 1729, 1749, 1751, 1759, 1762, 1764, 1766, 1770, 1772 y, tal vez, 1775. De este modo, en la tercera expedición remitida por la casa de Bassano se añadían, junto a la mercancía solicitada por Josep Bro, tres catálogos, posiblemente los del año 1772, que incluían la novedad de dedicar un volumen exclusivo para las estampas y los papeles decorados. Así, las guías de referencia para el último pedido de Josep Bro fueron el Catalogus novissimus et copiosissimus librorum omnium qui impressi sun, et vendutur apud Josephum Remondini et filios... y el Catalogo delle stampe in rame e delle varie qualità di carte.... En estos catálogos, de más volumen en cada nueva edición porque referenciaban un mayor surtido de productos a la vez que actualizaban los precios, se advertía a los corresponsales sobre la necesidad de no emplear para los pedidos los repertorios impresos en los años precedentes (Clemente, 2013).

Con los libreros españoles la casa Remondini no aceptaba como pago el intercambio de libros y, por consiguiente, exigía dinero en efectivo «pronti contanti». Los pagos, sin embargo, se podían realizar en un plazo comprendido entre los 12 y los 24 meses a contar desde el día de la partida de la expedición. Esta laxitud en la entrega del dinero resultaba muy conveniente para los corresponsales, pues de esta manera disponían de un margen de tiempo para colocar la mercancía y reunir, sino todo, al menos parte del capital que adeudaban.

Los intereses de la casa Remondini en Cataluña y Aragón eran administrados y defendidos por el comerciante barcelonés Oleguer Verdaguer y Ros desde finales de la década de los sesenta. Verdaguer se encargaba de repartir los catálogos entre los libreros, de recibir y comprobar la mercancía de los Remondini cuando 
era desembarcada en el puerto de Barcelona, de cobrar los adeudos pendientes de los libreros y de remitir las cantidades que iba percibiendo a través de Gaetano Gervasoni, cónsul de Venecia en Génova. Así mismo, mantenía una fluida correspondencia comercial con los editores de Bassano, a través de la cual daba cuenta de sus gestiones y recibía instrucciones sobre la manera de proceder contra los morosos.

Aunque Josep Bro recibió la primera remesa de material impreso y de papelería en 1764 no realizó su primer pago — parcial — hasta poco antes de formalizar su segundo pedido. El 1 de febrero de 1768 hizo llegar a Oleguer Verdaguer 735 libras venecianas. Esta entrega, unida a las 1.046 libras que remitió el 24 de octubre del mismo año sumaban un total de 1.781 , cantidad que no cubría el importe de la primera factura.

La segunda factura, de noviembre de 1768, ascendía a 1.675 libras, pero Josep Bro puso en evidencia algunos errores que se habían cometido al confeccionarla. En primer lugar, los 18 ejemplares del segundo tomo en $4^{\circ}$ de la obra de Fulgenzio Cuniliati, Universae theologiae moralis, impresos en la casa veneciana de Tommaso Bettinelli, habían sido contados a 18 libras venecianas el volumen, cuando en el catálogo estaba tasado en 12. Las 324 libras que figuraban en la factura tendrían que haber sido 216. Como el propio Bro observaba, seguramente se trataba de «una equivocación de su mayordomo» ${ }^{7}$, pero la diferencia suponía 108 libras de más. La reclamación que realizó Josep Bro por carta se atendió parcialmente: los Remondini establecieron el precio de los Cuniliati en 13 libras y 4 sueldos (237 libras y 12 sueldos por los 18 tomos) y, en abril de 1769, le aplicaron a su deuda una bonificación de 86 libras y 8 sueldos por la diferencia. El librero gerundense no quedó satisfecho con el arreglo y continuó interpelando por carta a los impresores venecianos para que atendiesen sus razones en este punto. En segundo lugar, protestó por el cargo de 41 libras venecianas por el seguro de mar desde Venecia hasta Génova, cuando la mercancía había viajado por tierra. A pesar de estas pequeñas diferencias sobre la segunda factura, el 19 de junio de 1769 Josep Bro entregó a Oleguer Verdaguer 400 libras venecianas - que correspondían a 75 libras catalanas. Tras este pago la deuda pendiente del librero gerundense con la casa Remondini era de 1.289 libras venecianas y 12 sueldos.

Como los requerimientos realizados por carta instando al pago no habían surtido efecto, el 27 de junio de 1771 los Remondini dieron poderes a Oleguer Verdaguer, su agente en Barcelona, para exigir al librero de Girona las 1.289 libras venecianas

ACA, Real Audiencia, Consulado y Tribunal de Comercio, C 6806, «Causa de José Remondiny... contra Bro» (1782). Carta de Josep Bro (Girona) a Josep Remondini (Venecia), 15 de febrero de 1775 . 
y 12 sueldos $^{8}$. No conocemos con exactitud las gestiones realizadas por Verdaguer para cumplir su cometido, pero todo parece indicar que llegó a un acuerdo con Bro para que éste le fuese entregando una cantidad de dinero en una fecha determinada de cada año hasta saldar la deuda. A través de la correspondencia que Oleguer Verdaguer envió a sus patrones de Bassano tenemos noticia de las pequeñas cantidades que iba recogiendo de Josep Bro, las cuales, sin embargo, no quedan especificadas ${ }^{9}$.

En abril de 1772, sin embargo, la casa Remondini tuvo que enfrentarse a una terrible acusación del gobierno de España. Una estampa, titulada «Juicio universal», que había sido confeccionada e impresa por la casa de Bassano a instancia de Louis Bonnardel, un comerciante francés afincado en Cádiz, fue considerada injuriosa a $\mathrm{Su}$ Majestad Carlos III, debido a que ubicaba el escudo real y el nombre del monarca más cerca del infierno que del cielo. Detrás de esta calumniosa estampa se vió la mano de los jesuitas expulsos en connivencia con el impresor de Bassano, lo cual provocó una contundente reacción diplomática de la corte de Madrid, que exigió a la República de Venecia actuar contra los Remondini. El gobierno veneciano sometió a Giovanni Battista Remondini a dos procesos que, sin embargo - y a pesar de las presiones del gobierno de Carlos III-, lo exculparon de los cargos que se le imputaban (Antón, 2009).

Este asunto, que mantuvo en vilo el negocio de los Remondini hasta el verano de 1773 y prácticamente interrumpió la relación epistolar con los corresponsales, se vió agravado con la muerte de Giovanni Battista Remondini el 7 de julio. Giuseppe Remondini se hizo cargo del negocio y envió una circular a todos los corresponsales a los cuales encarecía para que cotinuasen confiando en la nueva dirección del negocio al tiempo que los animaba a realizan pedidos, «copiosi e frequenti» ${ }^{10}$.

Cuando todavía estas contrariedades mantenían en vilo a la casa Remondini, Josep Bro, que juzgaría que sus entregas regulares de dinero a Oleguer Verdaguer eran suficientes como garantía de su compromiso de pago, realizó el 30 de junio de 1773 su tercer pedido. La empresa de Bassano, una vez superado con éxito su enfrentamiento con la corte de España y sobrepuesta de la muerte de Giovanni Battista, estaba deseosa de recuperar su actividad habitual y reestablecer los tratos con sus corresponsales, los cuales habían quedado desatendidos durante un año. En este particular contexto llegó a Venecia la comanda de Bro, la cual fue bien acogida y fue

Archivio di Stato di Vicenza (ASVi), Atti Notarili, not. Giovanni Mimiola (notario de Bassano entre 1767-1800), busta 414, protocolo 3.

MCBG-AR, 9, Carta de Oleguer Verdaguer a Remondini, Barcelona, 12 de junio de 1772; Id., 15 de mayo de 1773; Id., 31 de julio de 1773.

10 MCBG-AR, 9, Circular remitida por la casa Remondini a sus corresponsales, s.1., s.f. (probablemente, en Venecia o Basano, finales de julio de 1773). 
servida a finales del verano de aquel año. El coste de las mercancías solicitadas era de 2.113 libras venecianas, las cuales, sumadas a la deuda que arrastraba, ampliaban el débito a 3.402 libras venecianas y 12 sueldos.

Por parte de los impresores de Bassano continuaron los apremios para que Josep Bro satisfaciera la deuda. Sabemos que el 30 de diciembre de 1774 remitieron a Girona dos cartas en las que instaban a Bro a pagar sin más dilación. El librero gerundense, sin embargo, continuaba quejándose de algunos cargos en las facturaciones - particularmente de la equivocación en el precio de los Cuniliatis de la segunda factura - y, sobre todo, exponía la coyuntura económica que le impedía disponer de liquidez. Josep Bro se había entregado en los últimos años a la compra de bienes raíces y a la mejora de las fincas adquiridas. En Sant Gregori, una población a seis kilómetros distante de Girona, compró en 1771 una tierra boscosa sita en el lugar de Cartellà y la masía Parés — con sus tierras de labor ${ }^{11}$ —; en 1772 compró una tierra boscosa en el lugar de Sant Grau'12; y, en 1775, una pieza de tierra de cultivo en el lugar de Constantins ${ }^{13}$. En estas propiedades se invirtieron también muchos caudales en la construcción de un ribazo ${ }^{14}$, la fabricación de un pozo ${ }^{15}$, el levantamiento de una pared de piedra ${ }^{16}$, entre otras muchas mejoras. Las inversiones que había ido realizando le habían supuesto, solo en los años 1773 y 1774, un desembolso de 2.400 libras catalanas ${ }^{17}$.

Este alarde patrimonial se completaba con una casa en la calle Ballesterías de Girona, donde se ubicaba su vivienda, el taller de imprenta y la librería. Incluso aseguraba que hubiera podido hacer frente a toda la deuda si «ubiesse podido cobrar de muchas partidas que difer[ente]s libreros me deven». Por todo ello Josep Bro pedía confianza a los Remondini: «le doy palabra que de oy adelante Vm. experimentará mi correspondencia y que le aré entregando quando pueda a fin de que Vmd. quede servido y que será contento de mi correspondencia y satisfación» ${ }^{18}$. Así mismo se comprometía a viajar en breve a Barcelona para entregar a Oleguer Verdaguer una partida de dinero.

\footnotetext{
11 Arxiu Històric de Girona (A.H.G.), Notaría de Girona 1, 1162, 25 mayo 1771, fs. 464-465v y 4 de julio de 1771, fs. 560-561v.

$12 \quad I d ., 1163,29$ de enero de 1772 , fs. $88-89 \mathrm{v}$.

$13 \quad I d ., 1166,21$ de marzo de 1775 , fs. $187-188 \mathrm{v}$.

$14 \quad$ Id., 1162,1 de junio de 1771 , fs. $484-484 \mathrm{v}$.

$15 \quad I d ., 1163,5$ de febrero de 1772 , fs. $128-128 \mathrm{v}$.

$16 \quad$ Id., 1163, 5 de abril de 1772, fs. 343-343v.

17 ACA, Real Audiencia, Consulado y Tribunal de Comercio, C 6806, «Causa de José Remondiny... contra Bro» (1782). Carta de Josep Bro (Girona) a Josep Remondini (Venecia), 15 de febrero de 1775 .

$18 \quad I d$
} 
En julio de 1775 los Remondini recibieron 788 libras venecianas, un gesto que permitió a Josep Bro realizar un cuarto pedido ese mismo mes. La deuda quedó reducida a 2.614 libras venecianas y 12 sueldos, pero cuando llegó la factura en octubre de ese año - 2.243 libras - el pasivo ascendió a 4.857 libras venecianas y 12 sueldos. Los Remondini, sin embargo, aplicaron en noviembre dos bonificaciones a facturas anteriores que dejaron la deuda en 4.756 libras venecianas y 12 sueldos, una crecida cantidad difícil de asumir por el librero gerundense.

Trancurrieron 1776, 1777 y 1778 sin pedidos y sin pagos, hasta que el primero de febrero de 1779 la casa Remondini libró una letra de cambio de 310 libras, 17 sueldos y 11 dineros (moneda veneciana) a favor de Gaetano Gervasone, cónsul veneciano en Génova, y contra Josep Bro, la cual, sin embargo, fue protestada por el librero de Girona. El protesto ocasionó un coste de 33 libras venecianas, las cuales se sumaron a la deuda pendiente. En total los Remondini reclamaban a Bro 4.789 libras venecianas y 12 sueldos —que equivalían a 898 libras catalanas ${ }^{19}$.

\section{El pleito interpuesto por la casa Remondini contra Josep Bro ante el Tribunal de la Junta de Comercio de Barcelona}

Durante los años 1779 y 1780 los impresores venecianos trataron en vano de cobrar la cantidad que les adeudaba Josep Bro. Ante la inoperancia que había mostrado Oleguer Verdaguer y Ros en la defensa de sus negocios en Cataluña, decidieron confiar la recaudación de las cantidades pendientes de pago a Pedro Benasso, comerciante de Barcelona. El 11 de septiembre de 1780 Giuseppe Remondini firmó los poderes ante el notario Giovanni Mimiola en favor del nuevo agente, el cual se puso en contacto con Josep Bro para consensuar una estrategia de pago $^{20}$. El representante de la casa de Bassano estableció un acuerdo mediante el cual Bro se comprometía a entregar 100 libras catalanas durante la Pascua de 1781 y, posteriormente, a ir pagando 40 libras catalanas cada año $-\mathrm{y}$ si fuera posible una cantidad mayorhasta saldar la deuda.

La casa de Bassano continuaba manteniendo una correspondencia directa con el librero de Girona, pero éste en esos momentos excusaba la puntual comprensión de las misivas por el hecho de ignorarar el idioma italiano. Según argüia Bro, en Girona sólo un religioso tenía conocimiento de la lengua italiana y, como éste siempre estaba muy ocupado o no le venía bien, resultaba difícil encontrar un momento para que

19 Id., Exposición escrita realizada por Tomás Vinyals y Tos, procurador de la casa Remondini, de los hechos que justifican la demanda de los impresores de Bassano ante el Tribunal de la Junta de comercio de Barcelona, 4 de febrero de 1783.

20

ASVi, Atti Notarili, not. Giovanni Mimiola, busta 414, protocolo 3. 
tradujese las cartas. En una de ellas, fechada en Girona el 18 de enero de 1781, Josep Bro advertía a Giuseppe Remondini que «siempre e tenido gana y voluntad de satisfacer a Vm. lo que fuese justo y, al mismo tiempo, lo que pudiese hir pagándole» ${ }^{21}$. Por consiguiente, las razones del atraso de la deuda eran, por un lado, el desacuerdo entre las mercancías entregadas y los precios exigidos en las facturas y, por otro lado, la incapacidad de Bro para hacer frente a una cantidad tan crecida en un contexto de adversidades.

Josep Bro - aseguraba - no vendía nada por causa de la guerra con Inglaterra $\mathrm{y}$, además, todavía no había podido recuperarse de las enormes pérdidas que había padecido con motivo de la inundación que experimentó la ciudad de Girona a mediados de noviembre de 1777. Como consecuencia de aquel desastre había perdido más de 1.200 libras catalanas y no había podido satisfacer a sus acreedores ${ }^{22}$. A pesar de todo, confiaba en su trabajo y en la posibilidad de negocio que le habría la reciente concesión real para imprimir la Gaceta dos veces a la semana ${ }^{23}$.

También solicitaba una rebaja de la deuda en atención a que muchos de los libros remitidos no se ajustaban a lo solicitado o esperado. Por ejemplo, en el último pedido la Historia ecclesiastica de Nöel Alexandre se había solicitado en folio pero, como en ese formato no se encontraba, se había enviado en cuarto y, además, con «mala letra»; por contra, las obras de Benedicto XIV — De sacrosancto missae sacrificio y De festis - las había pedido en cuarto y las habían enviado en folio, por lo que no las compraba nadie; y los libros de rezo no tenían las iniciales coloradas, como era costumbre y, según Bro, «acá no azen casso sin tener la letra colorada» ${ }^{24}$. Por estos entuertos que habían provocado tanto menoscabo en la venta Josep Bro solicitaba un descuento de cien libras. La rebaja finalmente fue aceptada por Pedro Benasso en una carta fechada el 31 de marzo de 1781, aunque esta gracia resultó efímera.

Las prerrogativas de Pedro Benasso se extinguieron pronto, aunque no sabemos exactamente por qué. Todo parece indicar que su compañía comercial —Pedro Benasso y Cía- quebró. El 21 de diciembre de ese año 1781 Giuseppe Remondini, ante el notario Mimiola, otorgó nuevos poderes a Esteve Guilla, otro comerciante de Barcelona, para cobrar todas sus deudas en Cataluña, de las cuales la más crecida era

$21 \quad$ ACA, Real Audiencia, Consulado y Tribunal de Comercio, C 6806, «Causa de José Remondiny... contra Bro» (1782). Carta de Josep Bro (Girona) a Josep Remondini (Venecia), 18 de enero de 1781.

$22 \quad I d$.

23 En 1778 la Gaceta pasó a editarse dos veces a la semana, apareciendo los martes y los viernes (Núñez de Prado, 2002, pag. 152).

24 ACA, Real Audiencia, Consulado y Tribunal de Comercio, C 6806, «Causa de José Remondiny... contra Bro» (1782). Carta de Josep Bro (Girona) a Josep Remondini (Venecia), 18 de enero de 1781. 
la que tenía que satisfacer Josep Bro ${ }^{25}$. Al día siguiente la casa Remondini escribió a Bro para que no pagase ninguna cantidad a Benasso y tratase con Guilla un nuevo sistema de pago.

Esteve Guilla encargó los tratos con Bro a un amigo mercero de Barcelona llamado Lorenzo Ensaldo, el cual se trasladó a Girona con motivo de la feria de San Narciso a finales de octubre de 1782. El 6 de noviembre parece que llegaron a un acuerdo, aunque éste estaba pendiente de la aprobación de Guilla. La ratificación, sin embargo, no se produjo. La casa Remondini había decidido actuar contra Bro de manera más resolutiva y, por ello, Esteve Guilla interpuso un pleito ante el Tribunal de Comercio de Barcelona en el cual, además de la deuda pendiente, se solicitaban daños y costas.

El 14 de diciembre de 1782 el propio Lorenzo Ensalzo se presentó en Girona de parte de Esteve Guilla y entregó a Josep Bro una carta de Giuseppe Remondini y las letras citatorias que le intimaban a personarse ante el tribunal de Barcelona. Bro lamentaba amargamente la decisión pues, según discurría, eso provocaría que «tanto yo como Vms. gastemos el dinero malamente con abogados, procuradores y escribanos y demás $\rangle^{26}$. En esos momentos, además, no podía aportar ninguna cantidad para enjugar su deuda. Se declaraba empeñado en la protección de sus propiedades en Sant Gregori, debido a que algunas de sus tierras estaban expuestas a las avenidas del río Ter.

Nada desvelaba, sin embargo, de los numerosos y dilatados litigios que había mantenido durante los últimos años y que le habían supuesto desembolsos significativos: en 1771, contra su cuñado Francesc Lanús, rector de Tossa ${ }^{27}$; entre 1775 y 1783 , contra Mateu Gri, presbítero de la catedral, y su sobrino Esteve Corominas y Gri, payés de Sant Gregori, por lindes y derechos ${ }^{28}$; y, en 1779, contra Francesc Massana, trabajador de Constantins, también por derechos de propiedad ${ }^{29}$.

Ante el ineludible apremio Josep Bro tuvo que trasladarse a Barcelona a principios de enero de 1783 para hacer frente a la nueva causa. Allí dio poderes a Antonio Cardeñas para que actuara como su procurador, pero, a pesar de que Bro «tenía mucho que deducir y alegar en d[ic] ha causa con que poder rebatir», para ahorrar costas

$25 \quad$ ASVi, Atti Notarili, not. Giovanni Mimiola, busta 414, protocolo 3.

26 ACA, Real Audiencia, Consulado y Tribunal de Comercio, C 6806, «Causa de José Remondiny... contra Bro» (1782). Carta de Josep Bro (Girona) a Josep Remondini (Venecia), 16 de diciembre de 1782 .

27 A.H.G., Notaría de Girona 1, 1162, 7 de julio de 1771, fs. 573-573v.

28 A.H.G., Notaría de Girona 1, 1162, 25 mayo 1771, fs. 464-465v.

29 A.H.G., Notaría de Girona 1, 1170, 25 mayo 1779, fs. 95-97. 
y «correr amistosamente», decidió finalmente $\operatorname{pagar}^{30}$. El 17 de mayo de 1783, en una escritura realizada ante el notario público de Barcelona, Josep Gualba y Roig, se llegó a un acuerdo y se evitó la prosecución del juicio. A las 898 libras catalanas que debía (4.789 libras venecianas y 12 sueldos) la casa de Bassano ratificó un descuento de 100 libras, aunque se le cargaron 40 libras de costas por los gastos soportados por los Remondini ante el Tribunal de Comercio. En total tenía que satisfacer 838 libras catalanas, las cuales se pagaron de la siguiente manera: 440 libras entregó Bro a Esteve Guilla en efectivo el mismo 17 de mayo; las restantes 398 libras se pagaron a razón de 100 libras cada año, siendo la última paga de 98 libras, entregándose la primera cantidad el 6 de noviembre de 1784 y las restantes en los años consecutivos. Los dineros se pagarían en la ciudad de Girona y se completaría el pago de la deuda el 6 de noviembre de 1787.

\section{Conclusiones}

Tras el pacto extrajudicial entre la casa Remondini y Josep Bro finalizó una relación comercial que había durado veinte años. Aunque los tratos entre ambos no consiguieron ser del todo fluidos, los pedidos realizados por el librero gerundense a la casa veneciana ponen en evidencia una serie de circunstancias, todavía poco conocidas, sobre las importaciones de material impreso a España durante la segunda mitad del siglo XVIII.

En primer lugar, la naturaleza de los libros demandados: los principales textos solicitados eran obras latinas de teología, de derecho canónico y de liturgia. A pesar de las dificultades que introdujo el auto de Curiel de 1752 para la importación de libros de coro, éstos continuaron llegando desde fuera gracias a la bondad de los precios y al prestigio de las ediciones que se realizaban en Amberes, Roma o Venecia. También se solicitaron libros latinos de medicina y filosofía, aunque en una proporción significativamente inferior respecto a los anteriores.

En segundo lugar, las estampas y los papeles decorados: aunque Josep Bro sólo solicitó estampas en el primer pedido, se pone en evidencia la capacidad de los impresores de Bassano para captar la devoción y la sensibilidad de la iconográfica local. Así, dentro del surtido de estampas de santos, una imagen del catálogo representaba a «San Narcisso», el patrón de la ciudad de Girona, adornado con el báculo de obispo y las típicas moscas que lo rodean (Infelise, 1990, p. 48). Respecto a los papeles decorados, un artículo de lujo que se puso de moda durante el reinado de Carlos III, casi todo venía de fuera, sobre todo de Francia e Italia, al menos hasta el 
establecimiento en Madrid de la Real Fábrica de Papeles Pintados (1786). Bro solicitó papeles xilográficos, marmoleados, salpicados, colorados, plateados o dorados, los cuales eran utilizados para encuadernar libros y forrar otros objetos.

En tercer lugar, la estrategia comercial que la casa Remondini aplicó en España: su ámbito de actuación prioritario eran los puertos del Mediterráneo desde los cuales se dirigían las mercancías a los libreros y estamperos que las habían solicitado. Uno de los libreros o una compañía comercial asentada en cada uno de estos puertos cuidaba de los intereses de los editores de Bassano, es decir, velaba por el desembarco y distribución de los fardos y cajas, el cobro de las deudas y la representación legal.

Finalmente, Josep Bro no es más que uno de los muchos corresponsales españoles que comerciaron con la casa Remondini de Bassano desde los años cuarenta del siglo XVIII hasta finales de la centuria. También recibieron mercancías de Venecia Louis Bonnardel, Giuseppe Mortuel, Manuel Espinosa de los Monteros, Claude Bertrand, Manuel Rodríguez Flores (de Cádiz); los hijos de Hidalgo y González de la Bonilla y los hermanos Berard (de Sevilla); Félix Casas Martínez, Francisco Martínez de Aguilar y Ramón Gravier (de Málaga); Francisco Benedicto y José Santiago Gómez (de Murcia); Joaquín Ibáñez (de Orihuela); Manuel Cavero Cortés y Santiago Mallén (de Valencia); Nicolás Villalba y José Alegría (de Salamanca); Jacinto Hernández (de Toledo); Joaquín Martínez (de Zaragoza); y José Carbonell (de Santa Cruz de Tenerife). En Cataluña, además de Josep Bro, también mantuvieron relaciones comerciales con los Remondini Isidre Aguasvivas, Tomás Piferrer, Jaume Osset, Carles Gibert y Tutó, Joan Massuet, Mateo Sterling y Pere Fullà (de Barcelona); y Cristóbal Escuder (de Lleida). La magnitud de la expansión de los editores de Bassano en España a lo largo del siglo XVIII es merecedora de mayor atención, al igual que la participación de otros impresores venecianos - como Pezzana o Baglioni-, sin olvidar que un volumen significativo de esas mercancías continuaban viaje hacia América. 


\section{Bibliografía citada:}

Antón Pelayo, Javier (1998), La herencia cultural. Alfabetización y lectura en la ciudad de Girona (1747-1807), Bellaterra, Universitat Autònoma de Barcelona.

Antón Pelayo, Javier (2009), "La 'Causa di Spagna'. Antijesuitismo, comercio de estampas y relaciones diplomáticas entre España y Venecia durante el reinado de Carlos III", Estudis. Revista de Historia Moderna, 35, pp. 221-258.

Antón Pelayo, Javier (2011), "La cultura jurídica del Barroco y la difusión de la literatura legal. La biblioteca del vicario gerundense Francesc Veray (1787)”, Tiempos Modernos. Revista Electrónica de Historia Moderna, 23. http://www. tiemposmodernos.org/tm3/index.php/tm/article/viewFile/265/318

Arias de SaAvedra Alías, Inmaculada (2003), "Libros extranjeros en la biblioteca del matemático Benito Bails (1731-1797)”, en Villar García, María Begoña y Pezzi Cristóbal, Pilar (eds.), Los extranjeros en España. Actas del I Coloquio Internacional, celebrado en Málaga del 28 al 30 de noviembre de 2002, Málaga, Universidad de Málaga, tomo II, pp. 125-137.

Arias de SAAVEdra AlíAs, Inmaculada (2017), “Libros y autores británicos en las bibliotecas privadas españolas del siglo XVIII", en Camarero Bullón, Concepción y Gómez Alonso, Juan Carlos (coords.), El dominio de la realidad y la crisis del discurso. El nacimiento de la conciencia europea, Madrid, Ediciones Polifemo, pp. 321-365.

BAs MARTín, Nicolás (2013), "Un soplo de aire fresco: libros franceses en los Catálogos del siglo XVIII de la librería Mallén de Valencia”, Revista General de Información y Documentación, 23, pp. 173-201. https://doi.org/10.5209/rev_ rgid.2013.v23.n1.41452

Bertrand, Gilles; Cayuela, Anne; Del Vento, Christian y Mouren, Raphaële (dirs.) (2016), Bibliothèques et lecteurs dans l'Europe Moderne (XVIIe-XVIIIe siècles), Ginebra, Droz.

Bonnant, Georges (1999), Le livre genevois sous l'Ancien Régime, Ginebra, Droz.

Bragado Lorenzo, Javier y CARo LóPez, Ceferino (2004), "La censura gubernativa en el siglo XVIII”, Hispania, 217, pp. 571-600. https://doi.org/10.3989/hispania.2004.v64.i217.188

CARnelos, Laura (2008), I libri da risma. Catalogo delle edizioni Remondini a larga diffusione (1650-1850), Milán, FrancoAngeli.

CARo lóPez, Ceferino (2004), “Censura gubernativa, Iglesia e Inquisición en el siglo XVIII”, Hispania Sacra, 56, pp. 479-511. https://doi.org/10.3989/hs.2004.v56. i114.132 
CARraro, Evalinda (2013), Strategia e organizzazione in un'impresa editoriale del '700: i Remondini da Bassano, Venecia, Università Ca'Foscari, tesi di laurea.

Clemente San Roman, Yolanda (2013), “Giuseppe Remondini: un ejemplo de venta de libros en la Italia del siglo XVIII", Pecia Complutense: Boletín de la Biblioteca Histórica de la Universidad Complutense, 19, pp. 1-48.

De los Reyes Gómez, Fermín (2000), El libro en España y América. Legislación y censura, siglos $X V$-XVIII, Madrid, Arco Libros, 2 vols.

Escolano de Arrieta, Pedro (1796), Práctica del Consejo Real en el despacho de los negocios consultivos, instructivos y contenciosos, Madrid, Viuda e hijo de Marín, vol. 1.

FontAine, Laurence (1994), Histoire du colportage en Europe, XVe-XIXe siècle, París, Albin Michel.

García Cuadrado, Amparo (2015), "Un francés entre los libreros murcianos del siglo XVIII", Revista General de Información y Documentación, 25-1, pp. 141155. https://doi.org/10.5209/rev_rgid.2015.v25.n1.48982

Guinard, Jacques (1957), « Le livre dans la Péninsule Ibérique au XVIIIe siècle. Témoignage d'un libraire français », Bulletin Hispanique, 59/2, pp. 176-198. https://doi.org/10.3406/hispa.1957.3527

Infelise, Mario (1990), I Remondini. Stampa e industria nel Veneto del Settecento, Bassano del Grappa, Ghedina \& Tassoti Editori.

Infelise, Mario y Marini, Paola (eds.) (1990), Remondini: un editore del Settecento, Milán, Electa.

InfELiSE, Mario (1991), L'editoria veneziana nel '700, Milán, Franco Angeli.

Infelise, Mario y MARInI, Paola (eds.) (1992), L'editoria del '700 e i Remondini: atti del Convegno, Bassano, 28-29 settembre 1990, Bassano del Grappa, Ghedina \& Tassotti.

INFELISE, Mario (2016), "Remondini”, Dizionario biografico degli italiani, http:// www.treccani.it/enciclopedia/remondini_\%28Dizionario-Biografico\%29/ [fecha de consulta: 19/05/2017].

LÓPEZ, François (1987). « Un aperçu de la librairie espagnole au milieu du XVIII siècle », en VV.AA., De l'alphabetisation aux circuits du livre en Espagne, XVIeXIXe siècles. Paris, CNRS, pp. 387-416.

LóPEZ, François (2003), "Geografía de la edición. El comercio interior y exterior”, en Infantes, Víctor; López, François y Botrel, Jean-François, Historia de la edi- 
ción y de la lectura en España, 1472-1914, Madrid, Fundación Germán Sánchez Ruipérez, pp. 338-347.

Mirambell Belloc, Enric (1988), Història de la impremta a la ciutat de Girona, Girona, Institut d'Estudis Gironins.

NúÑEZ De Prado, Sara (2002), “De la Gaceta de Madrid al Boletín Oficial del Estado", Historia y Comunicación Social, 7, pp. 147-160.

PÉLIGRY, Christian (1984), « Le marché espagnol », en Martin, Henri y Chartier, Roger (eds.), Histoire de l'edition française. Le livre triomphant: 1660-1830, París, Promodis, pp. 370-377.

PIWNIK, Marie-Hélène (1989), « Libraires français et espagnols à Lisbonne au XVIIIe siècle », en VV.AA., Livres et libraires en Espagne et au Portugal (XVIe-XXe siècles), París, CNRS, pp. 81-98.

Rey Castelao, Ofelia (2008), "El comercio de libros en la Galicia del Antiguo Régimen”, Obradoiro de Historia Moderna, 17, pp. 277-302.

Rueda Ramírez, Pedro (2012), "Libros venales: los catálogos de venta de los libreros e impresores andaluces (siglos XVII-XVIII)", Estudios Humanísticos. Historia, 11, pp. 195-222.

Rueda Ramírez, Pedro y Agustí, Lluís (coords.) (2016), La publicidad del libro en el mundo hispánico (siglos XVII-XX). Los catálogos de venta de libreros y editores, Madrid, Calambur. 